\title{
Highly efficient induction and long-term maintenance of multipotent cardiovascular progenitors from human pluripotent stem cells under defined conditions
}

\author{
Nan Cao ${ }^{1, *}$, He Liang ${ }^{1, *}$, Jijun Huang ${ }^{1}$, Jia Wang ${ }^{1}$, Yixiong Chen ${ }^{1}$, Zhongyan Chen ${ }^{1}$, Huang-Tian Yang ${ }^{1,2}$ \\ ${ }^{1}$ Key Laboratory of Stem Cell Biology, Institute of Health Sciences, Shanghai Institutes for Biological Sciences, Chinese Academy \\ of Sciences \& Shanghai Jiao Tong University School of Medicine (SJTUSM), Shanghai 200025, China; ${ }^{2}$ Shanghai Stem Cell \\ Institute, Shanghai Jiao Tong University School of Medicine (SJTUSM), Shanghai 200025, China
}

Cardiovascular progenitor cells (CVPCs) derived from human pluripotent stem cells (hPSCs), including human embryonic stem cells (hESCs) and human induced pluripotent stem cells (hiPSCs), hold great promise for the study of cardiovascular development and cell-based therapy of heart diseases, but their applications are challenged by the difficulties in their efficient generation and stable maintenance. This study aims to develop chemically defined systems for robust generation and stable propagation of hPSC-derived CVPCs by modulating the key early developmental pathways involved in human cardiovascular specification and CVPC self-renewal. Herein we report that a combination of bone morphogenetic protein 4 (BMP4), glycogen synthase kinase 3 (GSK3) inhibitor CHIR99021 and ascorbic acid is sufficient to rapidly convert monolayer-cultured hPSCs, including hESCs and hiPSCs, into homogeneous CVPCs in a chemically defined medium under feeder- and serum-free culture conditions. These CVPCs stably self-renewed under feeder- and serum-free conditions and expanded over $10^{7}$-fold when the differentiation-inducing signals from BMP, GSK3 and Activin/Nodal pathways were simultaneously eliminated. Furthermore, these CVPCs exhibited expected genome-wide molecular features of CVPCs, retained potentials to generate major cardiovascular lineages including cardiomyocytes, smooth muscle cells and endothelial cells in vitro, and were non-tumorigenic in vivo. Altogether, the established systems reported here permit efficient generation and stable maintenance of hPSCderived CVPCs, which represent a powerful tool to study early embryonic cardiovascular development and provide a potentially safe source of cells for myocardial regenerative medicine.

Keywords: human pluripotent stem cells; directed differentiation; progenitor maintenance; cardiovascular progenitor cells; chemically defined medium

Cell Research (2013) 23:1119-1132. doi:10.1038/cr.2013.102; published online 30 July 2013

\section{Introduction}

Cardiovascular diseases remain the leading cause of death worldwide. Due to the limited capability of heart tissue to regenerate, cardiac muscle loss in myocardial infarction (MI) or heart attack is largely irreversible. As a result, the heart pumps less efficiently, leading to the

\footnotetext{
*These two authors contributed equally to this work. Correspondence: Huang-Tian Yang

Tel/Fax: +86-21-63852593

E-mail: htyang@sibs.ac.cn

Received 5 March 2013; revised 5 May 2013; accepted 17 May 2013; published online 30 July 2013
}

development of heart failure after MI. Stem cell-based transplantation therapy aiming to repair injured heart tissue may substantially improve current cardiac therapies. However, the lack of suitable cell sources represents one of the major hurdles to overcome $[1,2]$. Human pluripotent stem cells (hPSCs), including embryonic stem cells (ESCs) and induced pluripotent stem cells (iPSCs), open a new era of opportunities for cardiovascular research, drug discovery, toxicology testing, and myocardial therapies due to their unlimited in vitro proliferation capacity and their ability to generate major cell types that form the heart $[3,4]$. Over the past decades, dramatic progresses have been made in the differentiation of hPSCs towards cardiovascular fate, especially into cardiomyo- 
cytes (CMs), smooth muscle cells (SMCs) and endothelial cells (ECs) [5-10]. However, the induction is timeconsuming (2-4 weeks) with variability among various hPSC lines in the yield and purity of generated tissue cells $[1,4]$. The tumorigenic potential of the residual undifferentiated cells also raises the safety concerns for the application of hPSC-derived cardiovascular cells [11]. More importantly, transplantation of hESC-derived CMs into the infarcted heart has only yielded transient and marginal benefits [12-14]. These limitations are possibly attributed to the limited proliferative capacity of differentiated CMs and the lack of blood vessel formation to supply oxygen and nutrients [15].

Heart development is a well-organized process that involves the sequential induction of mesoderm, multipotent cardiovascular progenitor cells (CVPCs) and functional derivatives [16]. CVPCs derived from hPSCs are committed and capable of differentiation into multiple lineages of the heart without teratoma-forming ability, and thus they offer an appealing alternative avenue for myocardial regeneration [15]. Transplantation of CVPCs derived from hESCs [17] and murine iPSCs [18] into the infarcted heart results in $31 \%$ and $39 \%-69 \%$ improvement of the heart function, respectively, reflected by the left ventricular ejection fraction index, which is more effective than transplantation of PSC-derived CMs (5\% - 10\%). Thus, hPSC-derived CVPCs likely hold tremendous promise for the regenerative therapy for heart diseases, and for the better understanding of stem cell biology and early embryonic cardiovascular development. However, to realize these application potentials, the establishment of proper methods for the efficient generation and stable maintenance of CVPCs derived from hPSCs is one of the prerequisites.

Induction of multipotent CVPCs from hPSCs by sequential treatment of up to 5 growth factors for 4-6 days reaches an differentiation efficiency of $10 \%-60 \%$ through modulating multiple signaling pathways including bone morphogenetic protein (BMP), fibroblast growth factor (FGF), Activin/Nodal, stem cell factor (SCF)/c-kit, vascular endothelial growth factor (VEGF), and Wnt pathways [5, 17, 19-21]. Recently, we have also found that activation of mitogen-activated protein kinase MEK-ERK $1 / 2$ pathway plays a critical role in ascorbic acid (AA)-induced increased yield of iPSC-derived CVPCs [22]. These findings indicate that a robust generation of CVPCs from hPSCs is likely practicable through manipulation of the key developmental signaling pathways. However, due to the complex nature and temporal modulation of these signaling pathways, the differentiation efficiency varies among different hPSC lines with distinct responses to the concentration and treatment time of applied growth factors. Thus, it is critical to determine the essential factors in the differentiation of hPSCs into CVPCs for the development of simple and reproducible methods that allow the efficient conversion of hPSCs into homogeneous CVPCs without cell sorting in defined feeder- and serum-free conditions. Moreover, although CVPCs have been identified by multiple markers [15], the developmental logic and molecular basis underlying their fate decisions of self-renewal vs differentiation are poorly understood. In addition, significant challenges remain in terms of the stable expansion of hPSC-derived CVPCs that retain long-term self-renewal capability and multi-lineage cardiovascular differentiation potential.

To address these issues, we generated an enriched population of multipotent CVPCs from monolayer-cultured hESCs or hiPSCs in a chemically defined medium within 3 days by modulating the key developmental signaling pathways. Moreover, the generated CVPCs stably self-renewed and expanded in feeder- and serum-free conditions when glycogen synthase kinase 3 (GSK3), BMP and Activin/Nodal pathways were simultaneously inhibited. These clonally expanded CVPCs were able to generate major cardiovascular cell types in vitro, yet lacked teratoma-forming ability. Our findings present a strategy to generate homogeneous and non-tumorigenic CVPCs for cell-based therapies and pharmacological tests in treating heart diseases, and provide new insights into the understanding of the fundamental processes involved in cardiovascular lineage specification and maintenance.

\section{Results}

BMP signaling activation combined with GSK3 inhibition and AA treatment is sufficient for the efficient conversion of hPSCs into enriched CVPCs

To induce hPSCs towards the cardiovascular fate, we recapitulated critical signaling pathways involved in mesoderm formation and subsequent cardiac patterning, including Wnt, GSK3, FGF, BMP and Activin/Nodal pathways [16], by using various growth factors and small molecules. A single-cell-based monolayer system was used to make the condition more uniform and a Rhoassociated coiled-coil kinase (ROCK) inhibitor Y27632 (Y) was added during the first day of differentiation to improve cell survival [23] (Figure 1A). After a series of screenings, we found that a medium containing BMP4 (25 $\mathrm{ng} / \mathrm{ml})$, AA $(50 \mu \mathrm{g} / \mathrm{ml})$ and GSK3 inhibitor CHIR99021 (CHIR, $3 \mu \mathrm{M}$ ) efficiently converted undifferentiated H9 hESCs into a homogeneous population within 3 days (Figure 1A), and induced the expression of SSEA1 (also known as CD15 or FUT4), a recently identified surface 


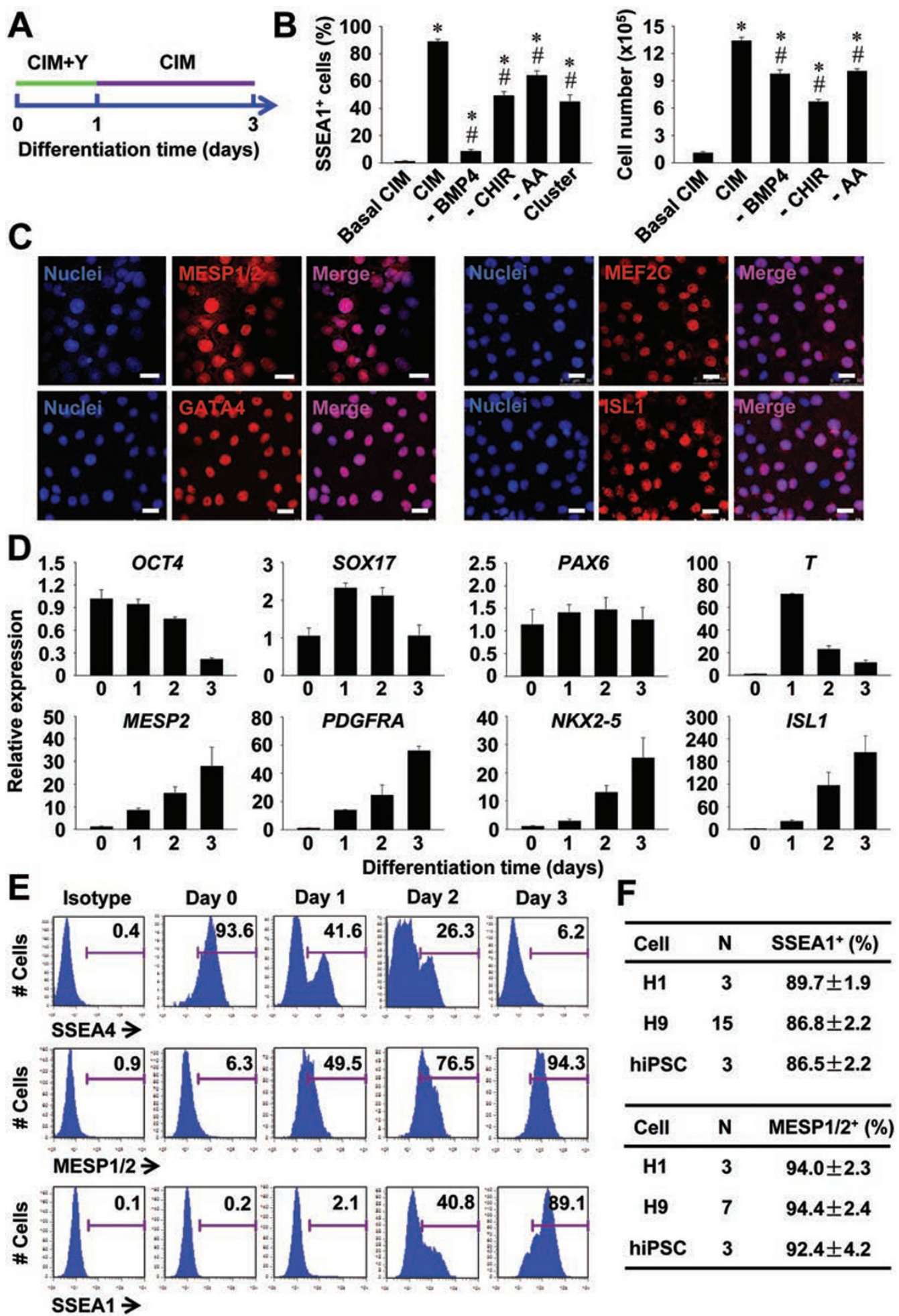

Figure 1 Induction of CVPCs from hPSCs. (A) An outline of the differentiation protocol. CIM, CVPC induction medium; Y, Y27632. (B) Percentage of SSEA $1^{+}$cells and total cell number at differentiation day 3 under various conditions $(n=3)$. CHIR, CHIR99021; AA, ascorbic acid. Basal CIM, CIM without CHIR, BMP4 and AA; - , indicates withdrawal. $* P<0.01$ vs Basal CIM; ${ }^{\#} P<0.01$ vs CIM. (C) Immunofluorescence analysis showing the expression of CVPC markers in cells at differentiation day 3 following CIM treatment. Scale bars $=25 \mu \mathrm{m}$. (D, E) qRT-PCR (D) and FACS (E) analysis showing the downregulation of pluripotency markers and upregulation of CVPC markers during CVPC induction $(n=3)$. (F) Induction efficiency of CVPCs from various $\mathrm{hPSC}$ lines. 
marker of human CVPCs [20, 24], in the majority (88.9\% $\pm 1.8 \%$ ) of the generated cells, with a yield of $4.8 \pm$ $0.4 \mathrm{SSEA}^{+}$cells per input hESC (Figure 1B). Therefore, this medium was named CVPC-induction medium (CIM) and used in the following studies. The withdrawal of BMP4, CHIR and AA from the CIM decreased the percentage of SSEA $1^{+}$cells by $90.3 \% \pm 1.6 \%, 44.5 \% \pm$ $4.3 \%$ and $27.7 \% \pm 5.0 \%$, respectively. In addition, seeding cells in clusters instead of single ones led to $49.4 \%$ $\pm 6.3 \%$ decreases of the percentage of SSEA $1^{+}$cells (Figure 1B, left panel). The total cell yield was also diminished by the withdrawal of BMP4, CHIR or AA from the CIM (Figure 1B, right panel). These results indicate that BMP4 signaling plays a key role in the generation of CVPCs from hESCs, and that AA treatment, which robustly activates the MEK-ERK1/2 pathway [22], together with GSK3 inhibition further promotes the induction process to efficiently convert hESCs into enriched CVPCs.

The hESC-derived cells exhibited homogeneous morphology during the differentiation process (Supplementary information, Figure S1A). Immunostaining analysis revealed that the cells at differentiation day 3 universally expressed the key CVPC marker MESP1/2, a sign for cells entering the cardiovascular fate [25], and other CVPC markers including MEF2C, GATA4 and ISL1 (Figure 1C), whereas none of these cells expressed the endodermal marker SOX17 (Supplementary information, Figure S1B). Stainings of the CVPC markers on the cells cultured in basal CIM (CIM without BMP4, CHIR and AA) were performed and excluded the possibility of nonspecific staining (Supplementary information, Figure S1C). Quantitative reverse transcription PCR (qRT-PCR) and fluorescence-activated cell sorter (FACS) analysis also confirmed that CIM treatment induced a rapid loss of the expression of pluripotency markers OCT4 (Figure 1D) and SSEA4 (Figure 1E) and a low and transient expression of endoderm marker $S O X 17$, but had no effects on the induction of neuroectoderm transcript $P A X 6$ (Figure 1D). In contrast, the expression of early mesodermal gene BRACHYURY-T (T) and CVPC-specific genes MESP2, PDGFRA, NKX2-5 and ISL1 was robustly upregulated (Figure 1D). Further, cell population expressing CVPC marker proteins SSEA1 and MESP1/2 was progressively increased during the differentiation process, and $\sim 90 \%$ of the cells were SSEA1-positive and $\sim 94 \%$ of the cells were MESP1/2-positive at differentiation day 3 (Figure 1E). A similar induction efficiency was observed for H1 hESC line and hAFDC-iPS-36 hiPSC line (Figure 1F). Taken together, the combination of BMP4, GSK3 inhibitor and AA allows a rapid and efficient conversion of hPSCs into homogeneous CVPCs in defined medium in a simple system.

Inhibition of BMP, Activin/Nodal and GSK3 pathways enables a long-term self-renewal of hPSC-derived CVPCs

The proliferation and lineage-commitment of CVPCs are regulated by the subtle developmental niche and complex hierarchy of multiple signal pathways including Wnt, FGF, BMP, Notch, Hedgehog (Hh), VEGF, platelet-derived growth factor (PDGF), MEK, retinoic acid (RA), and Activin/Nodal pathways $[1,16,20]$. Thus, it is challenging to renew and expand CVPCs ex vivo in defined conditions. We hypothesized that CVPCs can maintain self-renewal and persistently propagate if the differentiation-inducing cues are eliminated. To test this, we treated H9 hESC-derived CVPCs with nine chemical compounds that selectively inhibit critical signaling pathways related to CVPC specification (Supplementary information, Table S1), together with the GSK3 inhibitor CHIR that activates the canonical Wnt signaling pathway to stimulate CVPC proliferation [19]. The colony morphology of CVPCs was not observed when the CVPCs at differentiation day 3 were seeded into either the basal CVPC propagation medium (CPM) without any inhibitors (Figure 2A, left panel) or the medium containing a single inhibitor mentioned above (data not shown). Under these conditions, the cells rapidly differentiated and lost active proliferation capacity (data not shown). In contrast, CVPCs still formed round-shaped colonies and expanded for 7-8 passages when a pool of the 10 inhibitors were added in the culture medium (Supplementary information, Figure S2A), indicating a successful maintenance of the expandable CVPCs. However, under this condition, dead cells were easily observed in later passages, accompanied with a progressive cell degeneration (Supplementary information, Figure S2A, right panel).

To determine the contributions of these 10 inhibitors, we then serially removed individual inhibitors from the pool. The lack of the inhibitors of Notch, Hh, VEGF, PDGF and RA pathways in the pool did not affect the formation and propagation of CVPC colonies (data not shown). Further removing the inhibitors of FGF (SU5402) and MEK (PD0325901) did not alter the undifferentiated morphology of CVPC colonies, but significantly enhanced cell growth (Supplementary information, Figure S2B). Interestingly, addition of FGF2, a ligand of FGF/MEK pathway, did not further promote the CVPC propagation but rapidly induced CVPC differentiation (Supplementary information, Figure S2B, right panel), indicating a complex regulatory role of FGF signaling in CVPC proliferation and differentiation [16]. The CVPCs cultured in the medium containing GSK3 inhibitor 
CHIR, BMP inhibitor dorsomorphin (DOR) and Activin/ Nodal inhibitor A83-01 (the medium is named as CPM) stably self-renewed and retained a high-level expression of the CVPC marker MESP1/2 (Figure 2A). However, withdrawal of any of these inhibitors from the CPM led to an extensive initiation of loss of colony morphology, dramatic reduction of MESP1/2 expression (Figure 2A), and marked suppression of CVPC proliferation (Figure 2B). RT-PCR analysis also revealed the decreased expression of undifferentiated CVPC markers SSEA1 and $M E S P$ 2, along with the induction of the expression of differentiated cardiovascular marker $K D R$, cardiac markers TBX5, NKX2-5 and TNNT2, SMC markers ACTA2 and $M Y H 11$, and EC markers PECAM1 and CDH5 when any of these inhibitors was removed from the CPM (Figure $2 \mathrm{C}$ ). Together, these data demonstrated the essential role and minimal requirement of the co-inhibition of GSK3, BMP and Activin/Nodal pathways in the mainte- nance of CVPCs.

The CVPCs derived from hESC lines $\mathrm{H} 1$ and $\mathrm{H} 9$, and hiPSCs line hAFDC-iPS-36 stably propagated in the CPM for $>15$ passages, maintained homogeneous progenitor morphology and uniformly expressed primitive CVPC markers including SSEA1, MESP $1 / 2$ and ISL1 (Figure 3A and 3B). The maintained CVPCs were routinely split 1:3 and expanded over $10^{7}$-fold at passage 15 (Figure 3C). Population-doubling times of the H1- and H9-derived CVPCs at early (P3) and late passage (P15) were not significantly different, whereas it was slightly longer for hiPSC-derived ones at late passage (Figure 3D). Next, to determine whether the CVPC colonies that emerged in the CPM culture are clonal, we performed a cell dose response experiment [17]. The number of colonies developed showed a linear relationship with the starting number of cells plated for all three hPSC lines examined (Figure 3E), indicating that the CVPC colonies

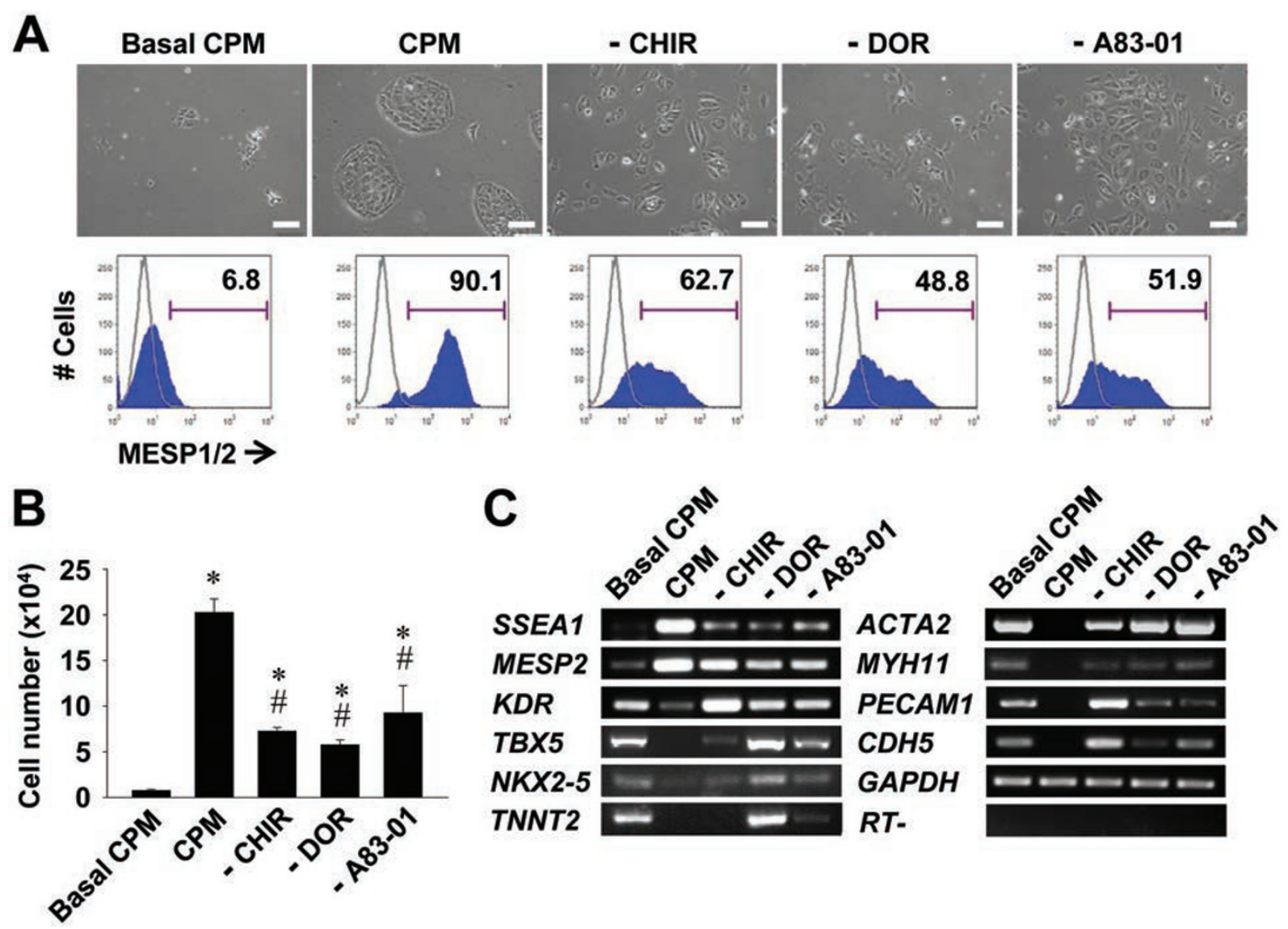

Figure 2 Conditions for CVPC maintenance. (A) Phase contrast images (upper panels); percentages of MESP1/2 cells (lower panels), (B) total cell numbers $(n=3)$, and $(C)$ the expression of CVPC, CM, SM and EC markers of H9 hESC-derived CVPCs at differentiation day 3 cultured in various conditions as indicated for another 5 days. CPM, CVPC propagation medium; DOR, dorsomorphin; Basal CPM, CPM without CHIR, DOR and A83-01. ${ }^{*} P<0.01$ vs Basal CPM; ${ }^{*} P<0.01$ vs CPM. 

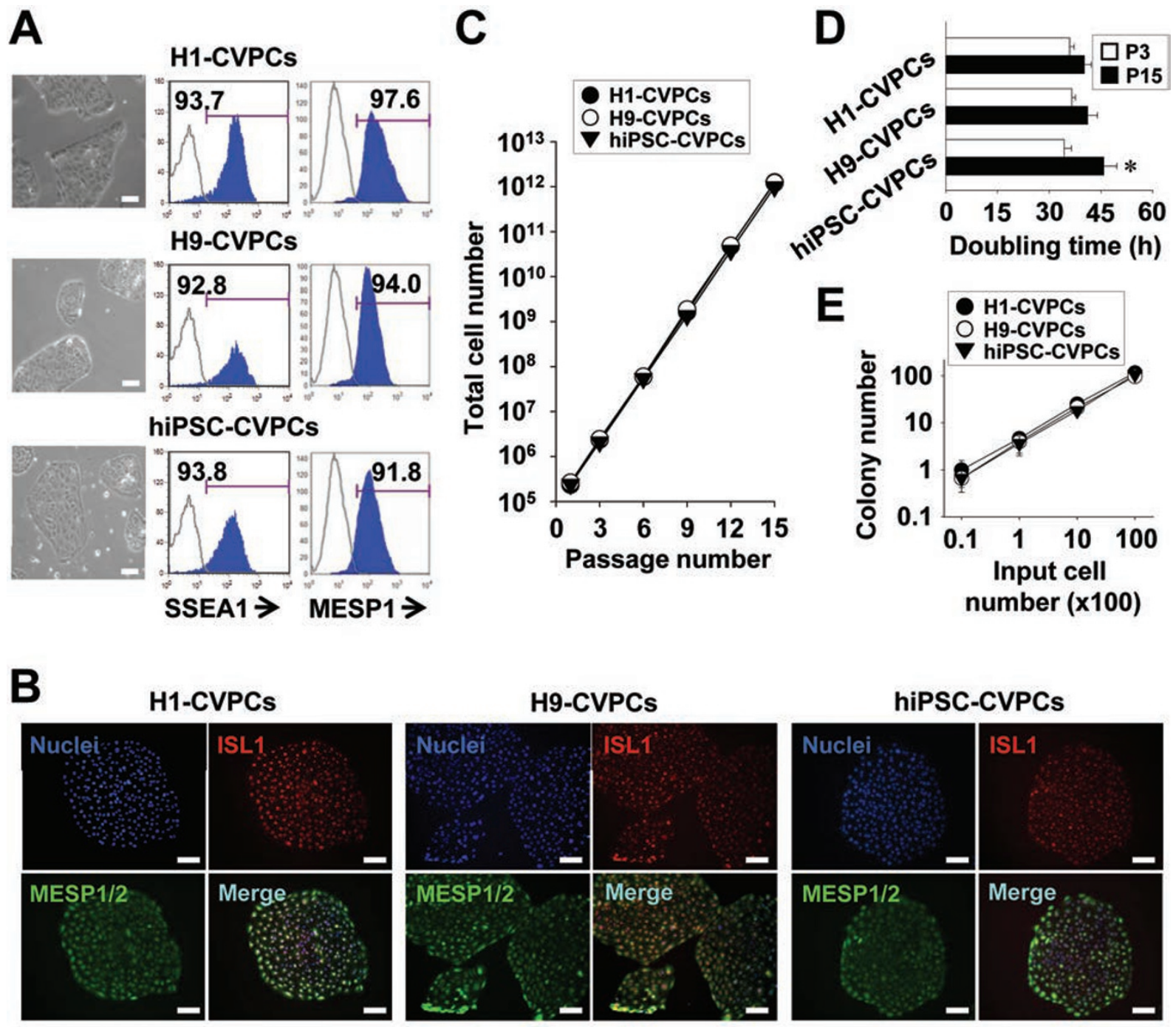

Figure 3 Long-term maintenance of hPSC-derived CVPCs. (A) Representative images showing the typical morphology (left panels), the SSEA1 and MESP1 expression analyzed by flow cytometry (middle and right panels). The control sample used in this assay was a 1:1:1 mixture from all three types of parental cells examined (H1-, H9-hESCs and hiPSCs). (B) Representative images of the ISL1 and MESP1 expression analyzed by immunostaining of CVPC colonies at passage 15. Scale bar $=100 \mu \mathrm{m}$. (C, D) Growth curves (C) and mean doubling time (D, $n=3)$ of CVPCs. ${ }^{*} P<0.05$ vs P3. (E) Cell dose response experiments showing the lineage relationship between the number of cells plated and the number of colonies developed $(n=3)$. Scale bars $=100 \mu \mathrm{m}$.

originate from single cells but not from cell aggregates.

\section{Genome-wide transcriptional profiling of CVPCs}

To understand the differences and similarities between the hPSCs and the freshly generated (P0) or long-term expanded (P15) CVPCs, we compared their transcriptomes by gene expression microarrays. Hierarchical cluster analysis of the differentially expressed genes with more than two-fold changes (Figure 4A) and scatter plot analysis of the global gene expression (Figure 4B) revealed that $\mathrm{P} 0$ and $\mathrm{P} 15$ CVPCs of either $\mathrm{H} 1$ or $\mathrm{H} 9$ origin shared a high degree of similarity in gene expression profiles, whereas they were clearly distinct from the hESCs cultured in basal CIM and undifferentiated hESCs. As expected, microarray analyses further confirmed the dramatic downregulation of pluripotency markers, accompanied by the significant upregulation of CVPC markers in both P0 and P15 CVPCs, compared with those in the hESCs and basal CIM-treated hESCs (Figure 4C). Moreover, the expression of endoderm- or ectoderm-specific genes was not significantly different among the cell types examined (Figure 4C). The expression of the markers of 


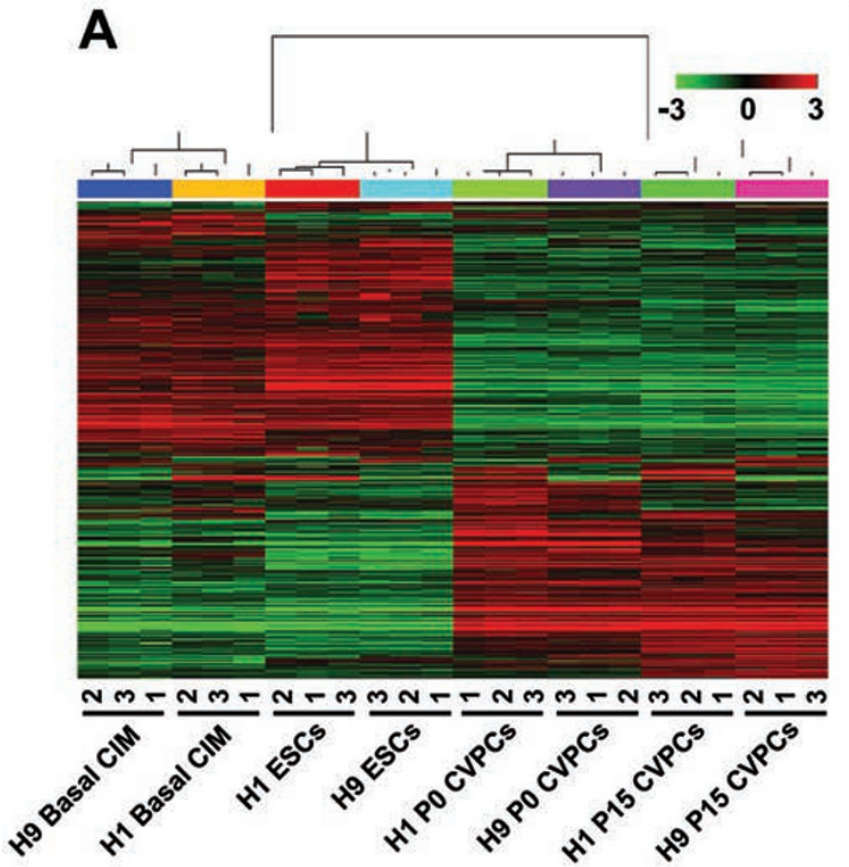

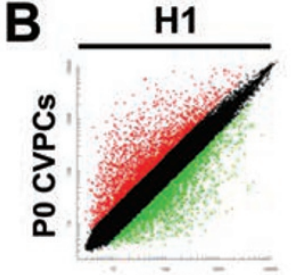

ESCs

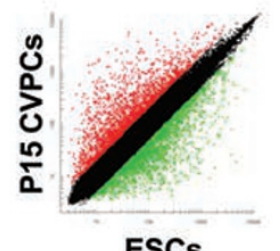

ESCs

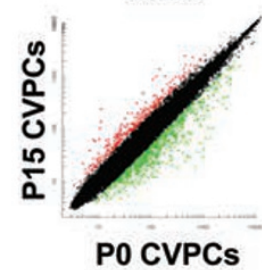

PO CVPCs \begin{tabular}{l|l} 
NANOG & \\
POU5F1 & \\
TDGF1 & \\
SOX2 & \\
DPPA4 & \\
FOXD3 & \\
GDF3 & \\
DPPA2 &
\end{tabular} LEFTY1 GATA3 ${ }_{\mathrm{TMP}}^{\mathrm{BM} 4}$ HAND ISL1 BMP2 GATA6. PDGFRA HAND2

GATA4

MEF $2 C$

NKX2-5 PDGFRB SOX17 FOXA2

HNF1B

HNF1A

HNF $4 A$

OXA HHEX GSC NEUROD1 SOX1 OTX1 PAX6 GBX2 ASCL1 ASCL2 2 N

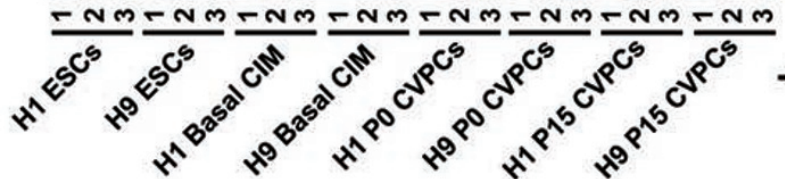

$\begin{array}{lll}-3 & 0 & 3\end{array}$

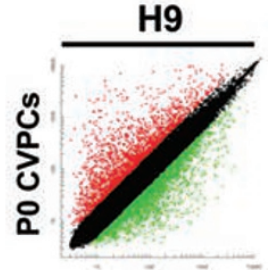

ESCs

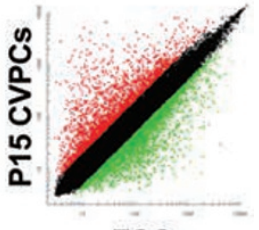

ESCs

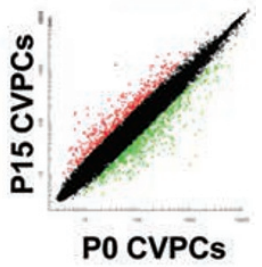

Figure 4 Genome-wide transcriptional profiling of CVPCs. (A, B) Hierarchical clustering (A) and scatter plot (B) analysis of global gene expression patterns in hESCs cultured in the basal CIM, ESCs, and CVPCs at passage 0 (P0) or 15 (P15), derived from either $\mathrm{H} 1$ or $\mathrm{H} 9 \mathrm{hESCs}$. The expression values in log2 scale in A were calculated and presented on the heat map with red representing upregulated genes and green representing downregulated genes. Black dots in B indicate the population with less than two-fold differences in gene expression levels between both samples. (C) Heat map showing expression levels of key marker genes related to pluripotency, CVPC, definitive endoderm, and neuronal ectoderm. 
primitive streak and early mesoderm (T, EOMES, MIXL1 and $L H X 1$ ), and hematopoietic progenitors (GATA1, $P T P R C, S P N$ and CD34) was also not detected in the CVPCs (microarray data not shown). These data indicate that the captured CVPCs derived from hPSCs display an expected CVPC genome-wide gene expression profile that is distinguished from that of the hPSCs.

\section{CVPCs lack tumorigenicity in vivo}

The propensity of hPSCs to form teratomas after transplantation remains a major safety concern in cellbased therapies. To determine whether the CVPCs generated in our study have tumorigenic potential, we intramuscularly transplanted P0 and P15 H9-derived CVPCs $\left(0.5-10 \times 10^{6}\right.$ cells $)$, and their parental H9 hESCs $(0.5 \times$ $10^{6}$ cells) into immune-repressed mice. All of the 4 mice injected with $\mathrm{H} 9 \mathrm{hESCs}$ developed teratomas 4 weeks after the transplantation (Figure 5A and 5B), while none of the animals receiving CVPCs (18 mice for P0 or P15 CVPCs) showed sign of tumor formation after 15 weeks (Figure 5A and 5B). Thus, it appears that both the freshly generated and long-term expanded CVPCs are not tumorigenic.

CVPCs exhibit multi-lineage cardiovascular differentiation potentials in vitro

Next, we tested whether the CVPCs retain multi-lineage cardiovascular differentiation potential by assessing their ability to differentiate into three major cardiovascular lineages. Representative data are from H9 hESCderived CVPCs at passage 15, if not indicated. To induce cardiac specification, the CVPCs were seeded at a high density in cardiac differentiation medium 1 (CDM1), exposed to a combined treatment of BMP4 and Wnt antagonist IWR1 for 3 days, and then cultured in CDM2 for another 9 days (Figure 6A). Spontaneously beating CMs were first observed at differentiation day 5 of CVPCs (Supplementary information, Movie S1). The cardiac differentiation was robust, leading to the formation of synchronizedly beating cell sheet at differentiation day 12 (Figure 6B, left upper panel and Supplementary information, Movie S2). Immunostaining analysis showed the

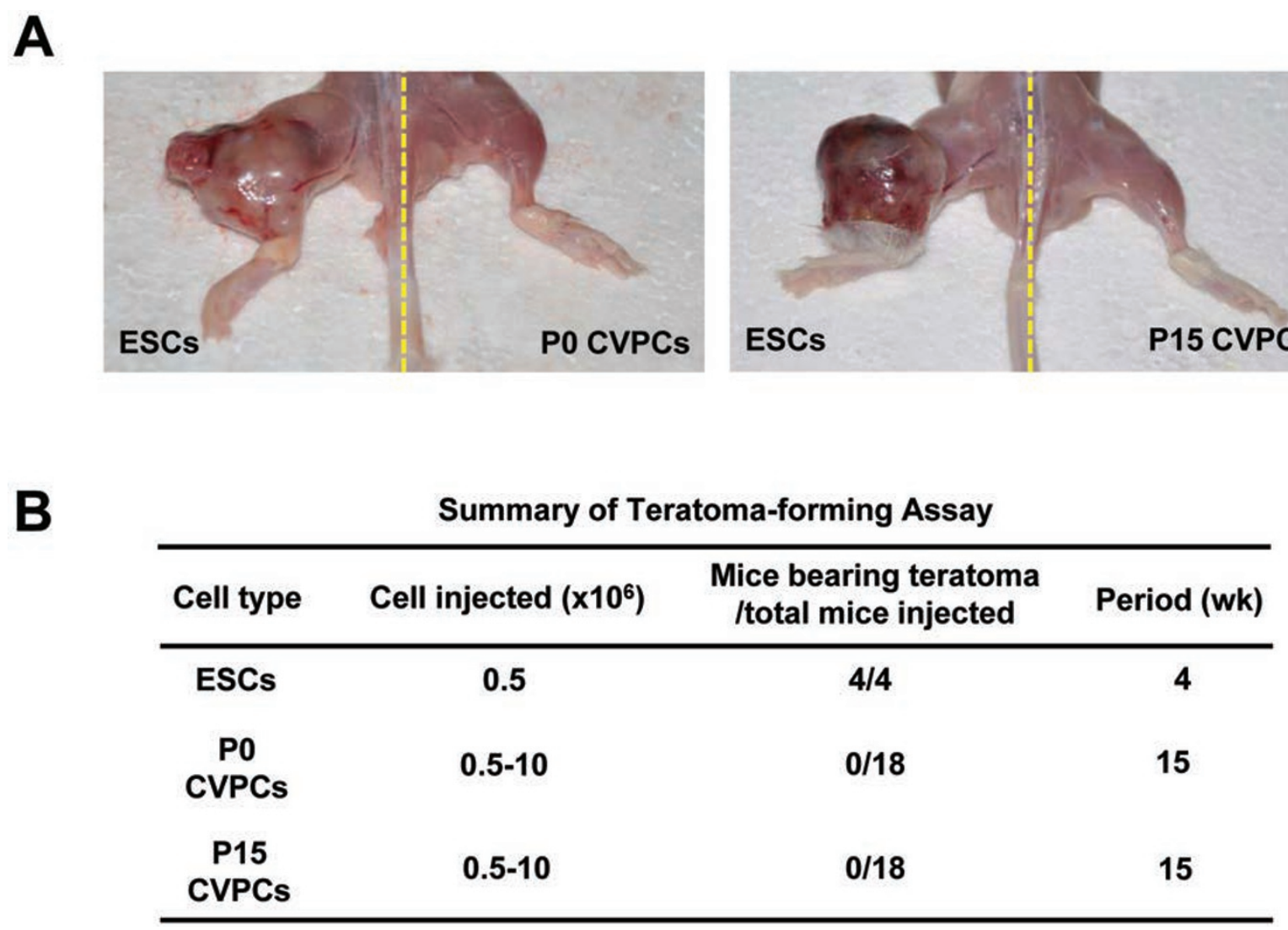

Figure 5 In vivo tumorigenicity analysis of CVPCs. (A) Representative images showing teratoma formation in left leg of the mouse injected with H9 hESCs (left panels) and lack of tumor formation in right leg of the mouse injected with P0 or P15 H9 hESC-derived CVPCs (right panels). (B) A summary of teratoma-forming ability of H9 hESCs and P0 or P15 CVPCs. 
A
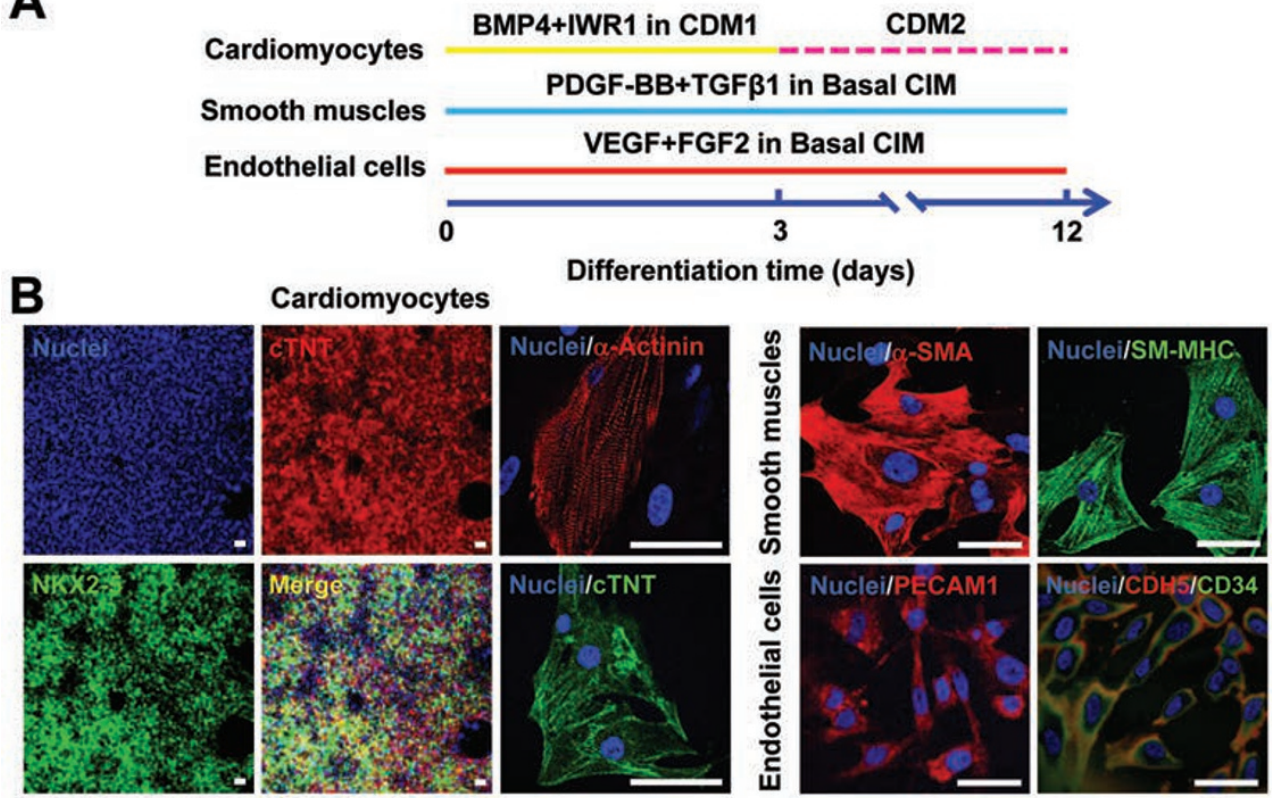

C
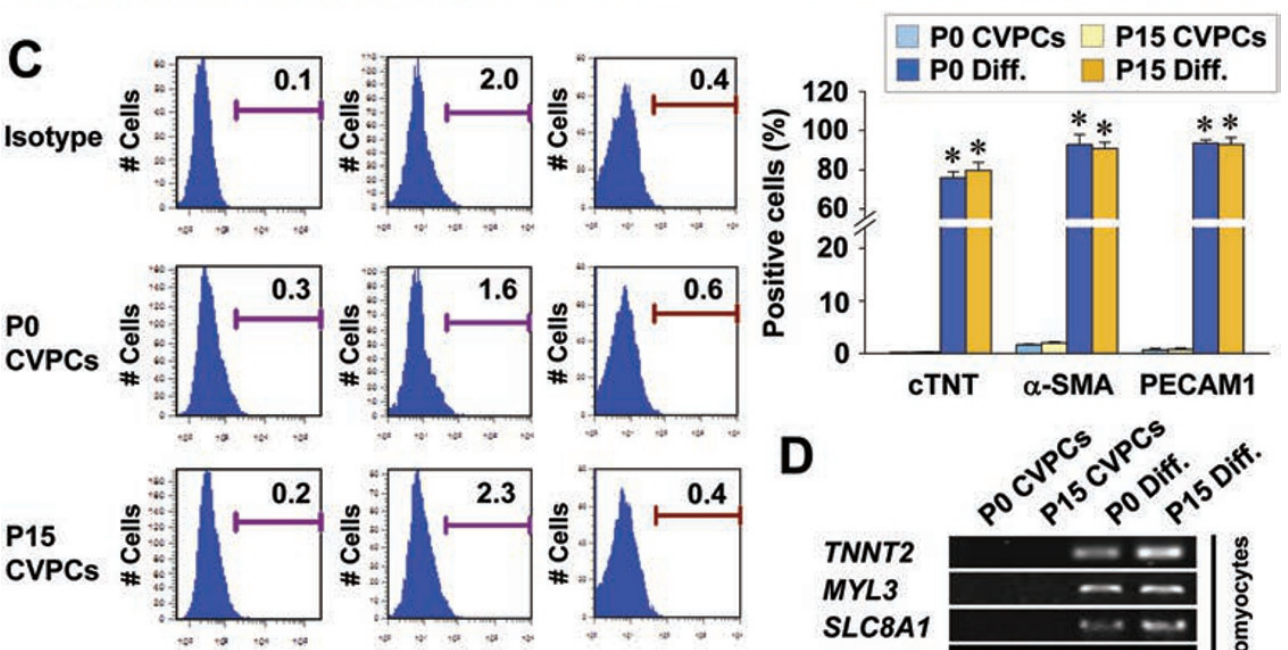

D

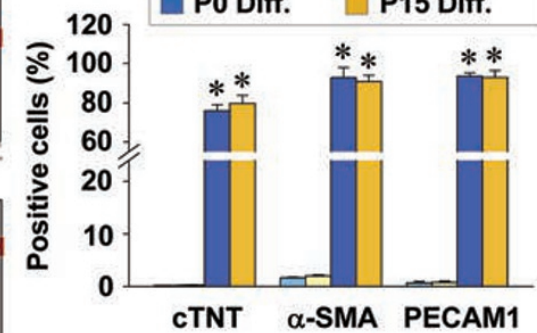

PO
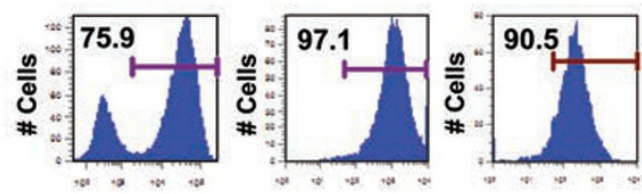

P15
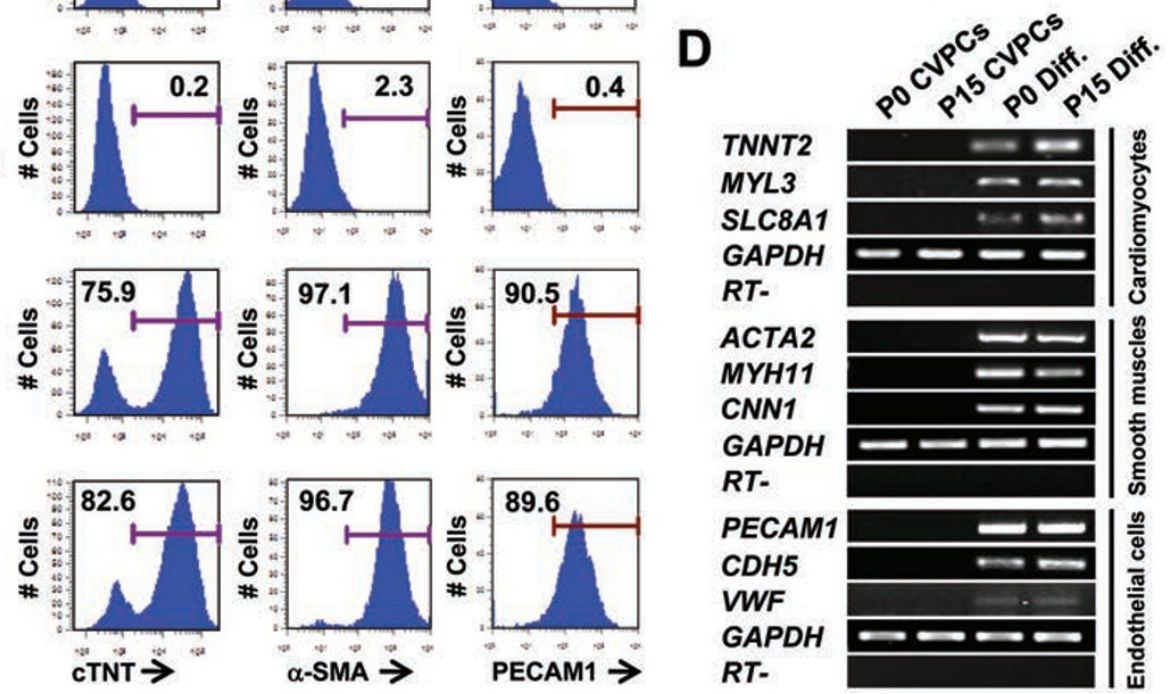

Figure 6 In vitro differentiation potential of CVPCs. (A) An outline of the conditions for inducing CVPC differentiation. (B) Differentiation potential of P15 CVPCs into cardiomyocytes, smooth muscle cells and endothelial cells determined by immunostaining analyses for NKX2-5 (green), cTNT (red or green), $\alpha$-Actinin (red) and merged NKX2-5 with cTnT in cardiomyocytes, $\alpha$-SMA (red) and SM-MHC (green) in smooth muscle cells, and PECAM1 (red), CDH5 (red), and CD34 (green) in endothelial cells after treatment in respective patterning conditions for 12 days. Scale bars $=50 \mu \mathrm{m}$. (C) Representative (left panels) and averaged FACS population analysis of $\mathrm{CTNT}^{+}$(CM marker), $\alpha-\mathrm{SMA}^{+}$(SMC cell marker) and PECAM $1^{+}(\mathrm{EC}$ marker) cells from $\mathrm{P0}$ and P15 CVPCs before or after treatment in respective patterning conditions for 12 days. $n=3$. $* P<0.01$ vs CVPC. (D) RT-PCR analysis showing the expression of cardiovascular derivate markers in various groups. 
coexpression of NKX2-5 and cTNT in CVPC-derived $\mathrm{CMs}$, and a clearly cross-striated pattern revealed by the staining of cardiac myofilamental proteins $\alpha$-Actinin and cTNT (Figure 6B, left panels), suggesting that the cells have developed organized sarcomeric structure.

To differentiate the CVPCs towards SMC or EC fate, we treated them with a combination of PDGF-BB and TGF $\beta 1$ (for SMCs), or VEGF plus FGF2 (for ECs) [7, 17, 20] (Figure 6A). Differentiated cells showed SMC or EC morphology, and expressed markers of SMCs ( $\alpha$-SMA and SM-MHC, Figure 6B, right upper panel) or markers of ECs (PECAM1, CDH5 and CD34, Figure 6B, right lower panel), indicating that CVPCs are capable of differentiation into the SMCs and ECs.

To evaluate the efficiency of CVPC differentiation into each cell type, the undifferentiated CVPCs at P0 and P15 with or without treatments of different lineage-patterning factors were examined by FACS analysis of specific lineage markers. The CM marker cTNT was expressed in $\sim 80 \%$ of the cells at differentiation day 12 (Figure 6C), with yields of $2.5 \pm 0.2$ (P0, $n=3$ ) and $2.5 \pm 0.4(\mathrm{P} 15$, $n=3) \mathrm{cTNT}^{+}$cells per input CVPC. About $90 \%$ of the cells were positive for SMC marker $\alpha$-SMA or EC marker PECAM1 when treated with the corresponding patterning cues (Figure 6C), although few cells positive for these markers were detected in untreated CVPCs (Figure $6 \mathrm{C}$ ). The yields of $\alpha-\mathrm{SMA}^{+} \mathrm{SMCs}$ were $3.3 \pm 0.1$ (P0, $n$ $=3)$ and $3.0 \pm 0.3(\mathrm{P} 15, n=3)$ per input CVPC, while the yields of PECAM1 ${ }^{+}$ECs were $1.7 \pm 0.2(\mathrm{P} 0, n=3)$ and $1.8 \pm 0.2(\mathrm{P} 15, n=3)$ per input CVPC. Moreover, no significant differences were observed in the differentiation efficiency towards $\mathrm{CMs}$, SMCs and ECs between P0 and P15 CVPCs (Figure 6C). These results were further consolidated by the RT-PCR analyses of the expression of cardiac markers TNNT2, MYL3 and SLC8A1, SMC markers $A C T A 2, M Y H 11$ and $C N N 1$, and EC marker genes $P E$ $C A M 1, C D H 5$ and $V W F$ in P0 and P15 CVPCs and their derivatives (Figure 6D). These results demonstrate that the hPSC-derived CVPCs possess high and multi-lineage cardiovascular differentiation capacity in vitro even after long-term expansion.

\section{Discussion}

The application of hPSCs requires their in vitro differentiation into a homogeneous cell population, especially multipotent and proliferable progenitors. Recent pioneer studies have successfully generated CVPCs from hPSCs by using embryoid body formation in the presence [19] or absence of serum [5, 17], or by using monolayer culture on feeder cells $[20,24]$. We here further extend these findings by the development of a chemically defined medium that rapidly and uniformly converts mono- layer hPSCs into non-tumorigenic CVPCs without cell sorting under feeder- and serum-free culture conditions. To our knowledge, this is the fastest and most efficient method so far to produce an early population of CVPCs from hPSCs. The CVPCs generated in our system show unique coexpression of multiple CVPC-related markers, including relatively earlier markers MESP $1 / 2$ and SSEA1, and markers with later expression peak such as ISL1, GATA4 and MEF2C during cardiac development and differentiation $[15,16]$. This expression pattern has been maintained in the long-term cultivation (Figure $3 \mathrm{~A}$ and $3 \mathrm{~B}$ ). Interestingly, both earlier and later CVPCrelated markers are coexpressed in the CVPCs generated in our system. Such phenomenon was also observed in some CVPC populations identified by previous studies $[20,26-28]$. It was also found that during the induction of CVPCs from mouse ESCs, Isl1 is coexpressed together with Mesp1 in $~ 50 \%$ of early Mesp1-expressing cells at differentiation day 4 [28]. Thus, our findings support the view that the markers thought to respectively represent earlier or later stage may show an overlapped expression pattern [26, 29-31]. Moreover, a most recent publication has shown that Mesp1-expressing cells that are typically regarded as the earliest cardiovascular progenitors also develop into hematopoietic and skeletal lineages in a context-dependent manner in mice [32]. These results raise the concern that whether the MESP $1 / 2^{+}$ cells described in this study also possess such ability to generate non-cardiovascular lineages, or represent mesodermal progenitor cells. It is currently unclear whether the function and regulatory mechanism of MESP $1 / 2$ are conserved between mouse and human. Considering the fact that the CVPCs described here not only express MESP $1 / 2$, but also highly express ISL1 and MEF2C, which are bona fide markers for the cells entering cardiovascular fate $[1,19,33]$, and their robust differentiation ability into major cardiovascular cell types (Figure 6), the SSEA $1^{+} / \mathrm{MESP} 1 / 2^{+} / \mathrm{ISL}^{+}{ }^{+}$cells generated and maintained in our system are most likely an early intermediate population that patterns the cardiovascular fate. Future studies should be made to further test their differentiation potentials to other mesodermal lineages.

Another important finding of the study is the longterm expansion of these CVPCs in defined medium. For stem cell-based therapies, it is highly desirable to generate expandable sources of committed progenitors with multiple but lineage-restricted potentials to differentiate into various tissue cells of interest. If achieved, these populations can be carefully quality-controlled and serve as starting materials skipping tumorigenic hPSCs. Meanwhile, such a system can avoid the contamination of unwanted cell lineages and generate the cell types of interest more simply, rapidly and efficiently [15]. 
Thus, the maintenance of self-renewing CVPCs derived from hPSCs is a critical step towards this goal. Previous studies showed that Isl $11^{+}$CVPCs derived from mouse ESCs transiently expanded for 7 days when cocultured with the cardiac mesenchymal cells (CMCs), and these CVPCs rely on the Wnt ligands secreted from the feeder layer of CMCs $[34,35]$. Another study further identified FGF pathway as a downstream target of $\mathrm{Wnt} / \beta$-catenin signaling in CVPC propagation [36]. The $\mathrm{ISL}^{+} \mathrm{CVPCs}$ derived from hESCs also showed an enhanced proliferation capacity when cultured on Wnt3a-secreting feeders [19]. Moreover, the Nkx2-5 $5^{+}$CVPCs derived from mESCs were passaged for $>100$ population doublings on a layer of mitotically inactivated MEFs in the presence of high-level serum [37]. However, establishing a system that supports the long-term self-renewal of PSC-derived CVPCs under serum- and feeder-free conditions remains an important challenge.

We have identified the co-inhibition of GSK3, BMP and Activin/Nodal signaling pathways as the minimal requirement for the renewal of hPSC-derived CVPCs. CVPCs maintained in this condition can be expanded over $10^{7}$-fold. The method reported here is very straightforward and relatively simple to implement. These findings also indicate that the CVPCs can intrinsically self-maintain at a stable proliferating status in vitro as observed in vivo [38], if they are shielded from the core differentiation-inductive signals, which is reminiscent of the previous findings in the self-renewal of ESCs [39]. These observations suggest that stem cells, not only ESCs but also lineage-committed progenitors, may have an innate program for self-replication that does not rely on extrinsic self-renewal stimuli. Thus, the strategy of inhibition of key differentiationinducing cues or their downstream targets as we reported here may facilitate the capture/maintenance of other selfrenewing progenitor cells. Further tests of this hypothesis by genetic downregulation of specific targets of these pathways are valuable and need to be performed in the future. Notably, although extrinsic stimuli seem to be dispensable for CVPC propagation, we observed that the proliferation rate tends to decrease after repeated passaging (Figure 3D), perhaps due to the lack of proper mitogens. It is worthwhile to screen proper mitogenetic factors (e.g., activators of STAT3 and PI3K/AKT pathways) to further enhance the propagating capacity of CVPCs.

The ability of clonally expanded CVPCs to efficiently generate the three main cardiovascular lineages in vitro is promising. It will provide not only a safer and more efficient starting point for regenerative medicine, but also a powerful platform to dissect the onset of human cardiac development. However, it is currently unclear whether these cardiovascular derivates originate from a homoge- neous population or, alternatively, from the diverse subpopulations within the CVPCs. As the differentiation efficiency towards each cardiovascular lineage is very high $(>80 \%)$, it seems unlikely that each lineage is generated from distinct subtype of the CVPCs, but this issue needs to be verified by the clonal formation assay in the future experiments. Moreover, further studies should also be performed to examine the in vivo differentiation potential of the CVPCs reported here and to determine whether the transplantation of these cells could improve the cardiac performances in heart diseases such as myocardial infarction.

In summary, we establish conditions for efficient induction and long-term maintenance of the SSEA $1^{+} /$ MESP $1 / 2^{+} / \mathrm{ISL}^{+}{ }^{+}$progenitor cells derived from hPSCs in serum- and feeder-free conditions by modulating key signaling pathways. These cells uniformly exhibit molecular features of CVPCs and possess the ability to generate three major cell lineages of the heart, yet lack tumorigenic ability. The systems developed here provide new insights into understanding the regulation of selfrenewal and differentiation of CVPCs. Our study would also facilitate the investigation of molecular events initiating human cardiovascular specification and offer a potentially safer source for myocardial regenerative medicine.

\section{Materials and Methods}

\section{Human PSC culture and CVPC induction}

Human ESC lines H1 and H9 (WiCell) and hiPSC line hAFDCiPS-36 (generated from human amniotic fluid-derived cells via ectopic expression of human OCT4/SOX2/KLF4/C-MYC factors) [40], were routinely maintained in commercially available mTeSR1 media (Stem Cell Technologies) on Matrigel-coated plates (human ESCs qualified, BD Biosciences) according to manufacturer's instructions. For preparing the Matrigel-coated plates, one Matrigel solution aliquot $(2 \mathrm{mg})$ was immediately transferred into $23 \mathrm{ml}$ cold DMEM/F12. Then, $1 \mathrm{ml}$ diluted Matrigel was added into one well of a six-well plate and allowed to set for $30 \mathrm{~min}$ at $37{ }^{\circ} \mathrm{C}$ before use. Different batch of Matrigel may affect the induction efficiency and yield of CVPCs and should be tested before use. For CVPC induction, undifferentiated hPSCs at $80 \%-90 \%$ confluence were digested into single cells by Accutase (Stem Cell Technologies) and plated onto Matrigel-coated culture dishes at a density of $5 \times 10^{4}$ cells $/ \mathrm{cm}^{2}$ in CIM containing DMEM/F12, $1 \times$ B27 supplement (without vitamin A), 1\% L-Glutamine, 1\% penicillin/streptomycin, $400 \mu \mathrm{M}$ 1-thioglycerol (Sigma), $50 \mu \mathrm{g} / \mathrm{mL}$ AA (Sigma), $25 \mathrm{ng} / \mathrm{mL}$ BMP4, and $3 \mu \mathrm{M}$ GSK3 inhibitor CHIR9902 1 (Stemgent). The CIM without AA, BMP4 and CHIR99021 was defined as basal CIM and used as a control. To enhance cell viability, ROCK inhibitor Y27632 at $5 \mu \mathrm{M}$ (Calbiochem) was added during the first day of hPSC differentiation and was removed during the medium change after $24 \mathrm{~h}$ (Figure 1A). The monolayer-cultivated cells were harvested at differentiation day 3 for further examina- 
tion or passaging. All cytokines are of human source and were purchased from R\&D System, and all cultivation substances for cell cultures were from Invitrogen BRL, unless stated otherwise.

\section{Long-term maintenance of CVPCs}

To maintain the CVPCs, confluent CVPCs at differentiation day 3 were split 1:3 using Accutase and routinely passaged onto Matrigel-coated plates in serum-free CPM, containing DMEM/F12, $1 \times$ B27 supplement (without vitamin A), $1 \times \mathrm{N} 2$ supplement, $1 \%$ nonessential amino acids, $1 \%$ L-Glutamine, $1 \%$ penicillin/streptomycin, $0.1 \mathrm{mM} \beta$-mercaptoethanol, $400 \mu \mathrm{M}$ 1-thioglycerol (Sigma), $3 \mu \mathrm{M}$ CHIR99021, $2 \mu \mathrm{M}$ BMP inhibitor dorsomorphin (Sigma) and $0.5 \mu \mathrm{M}$ Activin/Nodal inhibitor A83-01 (Stemgent). The CPM without CHIR99021, dorsomorphin and A83-01 was defined as basal CPM. Different batch of Matrigel may affect the propagation efficiency of CVPCs and was tested before use. The medium was changed daily until the cells reached $80 \%-90 \%$ confluency, ready to passage. During the initial five passages, overnight treatment of $5 \mu \mathrm{M}$ Y27632 was also used to improve cell survival, but it was not required in the following passages.

\section{Differentiation of CVPCs}

Cardiac differentiation of CVPCs was initiated by seeding CVPCs into Matrigel-coated plates at a density of $4 \times 10^{5}$ cells $/ \mathrm{cm}^{2}$ in serum-free CDM1 containing RPMI1640, 1× B27 supplement (without insulin), 1\% L-Glutamine and 1\% penicillin/streptomycin, and supplemented with $10 \mathrm{ng} / \mathrm{ml} \mathrm{BMP} 4$ and $5 \mu \mathrm{M}$ Wnt antagonist IWR1 (Calbiochem). Cells were treated under this condition for 3 days and then cultured in CDM2 containing RPMI1640, 1× B27 supplement, 1\% L-Glutamine and 1\% penicillin/streptomycin for another 9 days.

For SMC or EC lineage differentiation, undifferentiated CVPCs were digested into single cells by Accutase and plated onto Matrigel-coated culture dishes at a density of $10^{4}$ cells $/ \mathrm{cm}^{2}$ in basal CIM and stimulated with $10 \mathrm{ng} / \mathrm{ml}$ PDGF-BB and $2 \mathrm{ng} / \mathrm{ml}$ TGF- $\beta 1$, or $50 \mathrm{ng} / \mathrm{ml} \mathrm{VEGF}$ plus $10 \mathrm{ng} / \mathrm{ml} \mathrm{FGF} 2$, respectively, for 12 days according to methods described previously, with some modifications $[7,17,20]$.

\section{$R T-P C R$ and $q P C R$}

Total RNA was prepared using an RNeasy Plus Mini Kit (QIAGEN) following the manufacturer's instructions and treated with DNase I (Promega) for $15 \mathrm{~min}$ to eliminate the potential contamination of genomic DNA. cDNA was generated by reverse transcription of total RNA ( $1 \mu \mathrm{g})$ using oligo $(\mathrm{dT})$ primer and ReverTra Ace reverse transcriptase (Toyobo). PCR was carried out using Taq DNA Polymerase (Takara). The PCR primers are listed in Supplementary information, Table S2. GAPDH was used as an internal control, and samples without reverse transcription were used as negative controls. qPCR was performed and analyzed by kinetic real-time PCR using the ABI PRISM 7900 system (Applied Biosystems) with SYBR Green Realtime PCR Master Mix plus (Toyobo) for relative quantification of the indicated genes. The transcript of GAPDH was used for internal normalization. The qPCR primers are listed in Supplementary information, Table S3.

\section{Flow cytometry analysis}

Cells to be examined were harvested and dissociated by Nonenzyme Cell Dissociation Buffer (Invitrogen). For direct flow cytometry, the samples were then stained for the presence of appropriate human membrane markers including PE-conjugated SSEA1 (1:20; eBioscience), PE-conjugated SSEA4 (1:100, BD Biosciences), and Alexa Fluor 647-conjugated PECAM1 (1:200, BD Biosciences). PE-conjugated mouse IgMs (for SSEA1, 1:20, eBioscience), PE-conjugated mouse IgG3 (for SSEA4, 1:100, BD Biosciences), and Alexa Fluor 647-conjugated mouse IgG2a (for PECAM1, 1:200, BD Biosciences) were used as isotype-matched negative control.

To detect the intracellular antigen, the cells were fixed and permeabilized by Foxp3 Staining Buffer Set (eBioscience), blocked by $5 \%$ FBS and incubated with primary antibody of MESP $1 / 2$ (1:100, Aviva Systems Biology), cTNT (1:100, Abcam) and $\alpha$-SMA (1:200, Sigma). 10\% normal goat serum (For MESP1/2, Vector Laboratories), and mouse IgGs (1:100 for cTNT and 1:200 for $\alpha$-SMA, BD Biosciences) were used as negative controls. DyLight 488- or 549-conjugated antibodies (Jackson ImmunoResearch) were used as secondary antibody. Cells were then analyzed and quantified by flow cytometry (FACStar Plus Flow Cytometer, BD Biosciences).

\section{Immunocytochemical staining analysis}

Immunostaining assays were performed according to the protocol described before [41]. Briefly, cells were fixed with $4 \%$ paraformaldehyde, permeabilized in $0.3 \%$ Triton X-100 (Sigma), blocked in 10\% normal goat serum (Vector Laboratories) and then incubated with primary antibodies against MESP1/2 (1:100, Aviva Systems Biology), MEF2C (1:100, Cell Signaling), GATA4 (1:300, Santa Cruz Biotechnology), ISL1 (1:100, Developmental Studies Hybridoma Bank), NKX2-5 (1:200, Santa Cruz Biotechnology), $\alpha$-Actinin (1:300; Sigma), cTNT (1:300; Abcam), $\alpha$-SMA (1:200, Sigma), SM-MHC (1:200, DakoCytomation), PECAM1 (1:200, R\&D System), CDH5 (1:100, R\&D System) and CD34 (1:200; Santa Cruz Biotechnology) in $4{ }^{\circ} \mathrm{C}$ overnight and detected by DyLight 488 - or 549-conjugated secondary antibodies. Nuclei were stained with Hoechst33258 (Sigma). 10\% normal goat serum (for MESP1/2, MEF2C, NKX2-5, CDH5 and CD34, Vector Laboratories), and mouse IgGs (1:300 for GATA4, 1:100 for ISL1, $1: 300$ for $\alpha$-Actinin, 1:300 for cTNT, and 1:200 for $\alpha$-SMA, 1:300 for SM-MHC, 1:200 for PECAM1, BD Biosciences) were used as negative controls. A Nikon TS100 fluorescence microscope or Leica TCS SP2 confocal laser-scanning microscope was used for slide observation and image capture.

\section{Teratoma formation assay}

P0 or P15 H9 ESC-derived CVPCs $\left(0.5-10 \times 10^{6}\right.$ cells, $\left.n=18\right)$, and their parental H9 ESCs $\left(0.5 \times 10^{6}\right.$ cells, $\left.n=4\right)$, mixed with 100 $\mu \mathrm{l}$ culture medium containing $10 \%$ Matrigel, were injected intramuscularly into the leg of 20 non-obese diabetic/severe combined immune deficient (NOD/SCID) mice. All animal experiments were conducted in accordance with the Guide for the Care and Use of Animals for Research Purposes and were approved by the Animal Care Committee of Shanghai Institutes for Biological Sciences.

\section{Microarray analysis}

Three replicates of each cell type including: (i) undifferentiated hESCs, (ii) basal CIM-treated hESCs, (iii) P0 CVPCs, and (iv) P15 CVPCs from either H1 or H9 hESC origin were prepared. Total RNA of each sample was extracted using RNeasy Plus 
Mini Kit (QIAGEN) following the manufacturer's instructions, and treated with DNAse I (Promega) for $15 \mathrm{~min}$ to eliminate the potential contamination of genomic DNA. Quality-assessed RNA samples were hybridized to Affymetrix GeneChip HumanGene 1.0 ST arrays (Affymetrix). Twenty-four raw data files generated by the Affymetrix scanner passed data quality control were further performed with RNA normalization through the Affymetrix expression console.

\section{Statistical analysis}

Data were presented as means \pm SEM. Statistical significance of differences was estimated by one-way ANOVA or Student's $t$-test by SigmaStat 3.5 software (Sigma). $P<0.05$ was considered significant.

\section{Acknowledgments}

This study was supported by grants from the National Basic Research Program of China (NBRPC, 2011CB965300, 2009CB941100 and 2010CB945603), the National Natural Science Foundation of China (31030050), National Science and Technology Project of China (2012ZX09501-001-001), and Research Programs of Chinese Academy of Sciences (XDA01020204, GJHZ1225). We thank Dr Ying Jin (Institute of Health Sciences) for kindly providing the hiPSC line hAFDC-iPS-36 and Wenzhao $\mathrm{Li}$ (Institute of Health Sciences) for technical assistance in the teratoma formation assay.

\section{References}

1 Burridge PW, Keller G, Gold JD, Wu JC. Production of de novo cardiomyocytes: human pluripotent stem cell differentiation and direct reprogramming. Cell Stem Cell 2012; 10:1628.

2 Mummery CL, Zhang J, Ng ES, et al. Differentiation of human embryonic stem cells and induced pluripotent stem cells to cardiomyocytes: a methods overview. Circ Res 2012; 111:344-358.

3 Blin G, Neri T, Stefanovic S, Puceat M. Human embryonic and induced pluripotent stem cells in basic and clinical research in cardiology. Curr Stem Cell Res Ther 2010; 5:215226.

4 Murry CE, Keller G. Differentiation of embryonic stem cells to clinically relevant populations: lessons from embryonic development. Cell 2008; 132:661-680.

5 Kattman SJ, Witty AD, Gagliardi M, et al. Stage-specific optimization of activin/nodal and BMP signaling promotes cardiac differentiation of mouse and human pluripotent stem cell lines. Cell Stem Cell 2011; 8:228-240.

6 Sone M, Itoh H, Yamahara K, et al. Pathway for differentiation of human embryonic stem cells to vascular cell components and their potential for vascular regeneration. Arterioscler Thromb Vasc Biol 2007; 27:2127-2134.

7 Cheung C, Bernardo AS, Trotter MW, Pedersen RA, Sinha $\mathrm{S}$. Generation of human vascular smooth muscle subtypes provides insight into embryological origin-dependent disease susceptibility. Nat Biotechnol 2012; 30:165-173.

8 Lian X, Hsiao C, Wilson G, et al. Robust cardiomyocyte differentiation from human pluripotent stem cells via temporal modulation of canonical Wnt signaling. Proc Natl Acad Sci USA 2012; 109:E1848-E1857.

9 Zhang J, Klos M, Wilson GF, et al. Extracellular matrix promotes highly efficient cardiac differentiation of human pluripotent stem cells: the matrix sandwich method. Circ Res 2012; 111:1125-1136.

10 Minami I, Yamada K, Otsuji TG, et al. A small molecule that promotes cardiac differentiation of human pluripotent stem cells under defined, cytokine- and xeno-free conditions. Cell Rep 2012; 2:1448-1460.

11 Hentze H, Soong PL, Wang ST, et al. Teratoma formation by human embryonic stem cells: evaluation of essential parameters for future safety studies. Stem Cell Res 2009; 2:198-210.

12 Laflamme MA, Chen KY, Naumova AV, et al. Cardiomyocytes derived from human embryonic stem cells in prosurvival factors enhance function of infarcted rat hearts. Nat Biotechnol 2007; 25:1015-1024.

13 van Laake LW, Passier R, Monshouwer-Kloots J, et al. Human embryonic stem cell-derived cardiomyocytes survive and mature in the mouse heart and transiently improve function after myocardial infarction. Stem Cell Res 2007; 1:9-24.

14 Cao F, Wagner RA, Wilson KD, et al. Transcriptional and functional profiling of human embryonic stem cell-derived cardiomyocytes. PLoS One 2008; 3:e3474.

15 Lam JT, Moretti A, Laugwitz KL. Multipotent progenitor cells in regenerative cardiovascular medicine. Pediatr Cardiol 2009; 30:690-698.

16 Noseda M, Peterkin T, Simoes FC, Patient R, Schneider MD. Cardiopoietic factors: extracellular signals for cardiac lineage commitment. Circ Res 2011; 108:129-152.

17 Yang L, Soonpaa MH, Adler ED, et al. Human cardiovascular progenitor cells develop from a KDR+ embryonic-stem-cellderived population. Nature 2008; 453:524-528.

18 Mauritz C, Martens A, Rojas SV, et al. Induced pluripotent stem cell (iPSC)-derived Flk-1 progenitor cells engraft, differentiate, and improve heart function in a mouse model of acute myocardial infarction. Eur Heart J 2011; 32:2634-2641.

19 Bu L, Jiang X, Martin-Puig S, et al. Human ISL1 heart progenitors generate diverse multipotent cardiovascular cell lineages. Nature 2009; 460:113-117.

20 Blin G, Nury D, Stefanovic S, et al. A purified population of multipotent cardiovascular progenitors derived from primate pluripotent stem cells engrafts in postmyocardial infarcted nonhuman primates. J Clin Invest 2010; 120:1125-1139.

21 Elliott DA, Braam SR, Koutsis K, et al. NKX2-5(eGFP/w) hESCs for isolation of human cardiac progenitors and cardiomyocytes. Nat Methods 2011; 8:1037-1040.

22 Cao N, Liu Z, Chen Z, et al. Ascorbic acid enhances the cardiac differentiation of induced pluripotent stem cells through promoting the proliferation of cardiac progenitor cells. Cell Res 2012; 22:219-236.

23 Watanabe K, Ueno M, Kamiya D, et al. A ROCK inhibitor permits survival of dissociated human embryonic stem cells. Nat Biotechnol 2007; 25:681-686.

24 Leschik J, Stefanovic S, Brinon B, Puceat M. Cardiac commitment of primate embryonic stem cells. Nat Protoc 2008; 3:1381-1387.

25 Bondue A, Blanpain C. Mesp1: a key regulator of cardiovascular lineage commitment. Circ Res 2010; 107:1414-1427. 
26 Moretti A, Bellin M, Jung CB, et al. Mouse and human induced pluripotent stem cells as a source for multipotent Isl1+ cardiovascular progenitors. FASEB J 2010; 24:700-711.

27 Nelson TJ, Faustino RS, Chiriac A, et al. CXCR4+/FLK-1+ biomarkers select a cardiopoietic lineage from embryonic stem cells. Stem Cells 2008; 26:1464-1473.

28 Bondue A, Tannler S, Chiapparo G, et al. Defining the earliest step of cardiovascular progenitor specification during embryonic stem cell differentiation. J Cell Biol 2011; 192:751-765.

29 Burridge PW, Thompson S, Millrod MA, et al. A universal system for highly efficient cardiac differentiation of human induced pluripotent stem cells that eliminates interline variability. PLoS One 2011; 6:e18293.

30 Bondue A, Lapouge G, Paulissen C, et al. Mesp1 acts as a master regulator of multipotent cardiovascular progenitor specification. Cell Stem Cell 2008; 3:69-84.

31 Uosaki H, Fukushima H, Takeuchi A, et al. Efficient and scalable purification of cardiomyocytes from human embryonic and induced pluripotent stem cells by VCAM1 surface expression. PLoS One 2011; 6:e23657.

32 Chan SS, Shi X, Toyama A, et al. Mesp1 patterns mesoderm into cardiac, hematopoietic, or skeletal myogenic progenitors in a context-dependent manner. Cell Stem Cell 2013; 12:587601.

33 Laugwitz KL, Moretti A, Caron L, Nakano A, Chien KR. Islet1 cardiovascular progenitors: a single source for heart lineages? Development 2008; 135:193-205.
34 Moretti A, Caron L, Nakano A, et al. Multipotent embryonic isl1+ progenitor cells lead to cardiac, smooth muscle, and endothelial cell diversification. Cell 2006; 127:1151-1165.

35 Qyang Y, Martin-Puig S, Chiravuri M, et al. The renewal and differentiation of Is11+ cardiovascular progenitors are controlled by a Wnt/beta-catenin pathway. Cell Stem Cell 2007; 1:165-179.

36 Cohen ED, Wang Z, Lepore JJ, et al. Wnt/beta-catenin signaling promotes expansion of Isl-1-positive cardiac progenitor cells through regulation of FGF signaling. J Clin Invest 2007; 117:1794-1804.

37 Christoforou N, Miller RA, Hill CM, et al. Mouse ES cellderived cardiac precursor cells are multipotent and facilitate identification of novel cardiac genes. J Clin Invest 2008; 118:894-903.

38 Cai CL, Liang X, Shi Y, et al. Isl1 identifies a cardiac progenitor population that proliferates prior to differentiation and contributes a majority of cells to the heart. Dev Cell 2003; 5:877-889.

39 Ying QL, Wray J, Nichols J, et al. The ground state of embryonic stem cell self-renewal. Nature 2008; 453:519-523.

40 Li C, Zhou J, Shi G, et al. Pluripotency can be rapidly and efficiently induced in human amniotic fluid-derived cells. Hum Mol Genet 2009; 18:4340-4349.

41 Cao N, Liao J, Liu Z, et al. In vitro differentiation of rat embryonic stem cells into functional cardiomyocytes. Cell Res2011; 21:1316-1331.

(Supplementary information is linked to the online version of the paper on the Cell Research website.) 